\title{
The comparison of hyaluronic acid vaginal tablets in the treatment of atrophic vaginitis
}

\author{
Arif Serhan Cevrioglu • Nermin Akdemir • \\ Gokcen Ilhan
}

Received: 25 September 2011/ Accepted: 5 January 2012/Published online: 25 January 2012

(c) Springer-Verlag 2012

\section{Dear Sir,}

We read the study titled "The comparison of hyaluronic acid vaginal tablets in the treatment of atrophic vaginitis: a randomized controlled trial" by Dr. Murat Ekin et al. published in Arch Gynecol Obstet (2011) 283:539-543 with interest. It is reported in the study that hyaluronic acid-containing vaginal tablets can be effective as an alternative treatment agent for the treatment of atrophic vaginitis in post-menopausal women who cannot use local estrogen. There are some issues which attracted our attention in the material methodology part.

It is reported in Table 3, where pre-treatment epithelial atrophy levels of the groups are compared, that about $1 / 4$ $(23.8 \%)$ of the women in the hyaluronic acid vaginal tablet group (group 2) had severe epithelial atrophy, while none of the cases included in the estradiol vaginal tablet group had severe epithelial atrophy. The authors showed in the table that a statistically significant difference ( $p$ 0.012) occurred in the epithelial atrophy signs of the groups in the basal examination. Similar proportional differences between the two groups are manifest in Table 4 where vaginal $\mathrm{pH}$ evaluation results are presented as well. Although it is indicated in the title that the study is a randomized trial, there is no explanatory information about the method by which the patients allocated to study and control groups were randomized.

The fact that the groups were not homogeneous in terms of vaginal epithelial atrophy signs before treatment suggests a failure of proper randomization in the allocation to study and control groups. The bias in the patient allocation at the beginning of the treatment will affect the evaluation of the treatment outcomes in the study and control group patients who are already small in number. In other words, the results may be expected to favor the group receiving estrogen, which had a better basal condition. We believe that we can make a better evaluation of the study results, if the authors would illuminate the aforementioned issues.

PS 1 We think that it is a typography error that the 20 patients in group 1 are written as $5.2 \%$ in Table 3 , although they correspond to $95.2 \%$ of the patients proportionally.

Conflict of interest The authors declare that they have no conflict of interest.
A. S. Cevrioglu $(\bowtie) \cdot N$. Akdemir · G. Ilhan

Department of Obstetrics and Gynecology, Sakarya University School of Medicine, Sakarya Research and Education Hospital, Sakarya, Turkey

e-mail: ascevrioglu@ hotmail.com 